\title{
Thiamine Deficiency-Induced Partial Necrosis and Mitochondrial Uncoupling in Neuroblastoma Cells Are Rapidly Reversed by Addition of Thiamine
}

\author{
L. Bettendorff, $*$ F. Sluse, $†$ G. Goessens, P. Wins, and T. Grisar \\ Laboratories of Neurochemistry and †Cellular and Tissular Biology, University of Liège, Liège; \\ and $*$ Laboratory of Bioenergetics, University of Liège, Sart-Tilman, Belgium
}

\begin{abstract}
Culture of neuroblastoma cells in a medium of low-thiamine concentration $(6 \mathrm{nM})$ and in the presence of the transport inhibitor amprolium leads to the appearance of overt signs of necrosis; i.e., the chromatin condenses in dark patches, the oxygen consumption decreases, mitochondria are uncoupled, and their cristae are disorganized. Glutamate formed from glutamine is no longer oxidized and accumulates, suggesting that the thiamine diphosphate-dependent $\alpha$-ketoglutarate dehydrogenase activity is impaired. When thiamine $(10 \mu \mathrm{M})$ is added to the cells, the $\mathrm{O}_{2}$ consumption increases, respiratory control is restored, and normal cell and mitochondrial morphology is recovered within $1 \mathrm{~h}$. Succinate, which is oxidized via the thiamine diphosphate-independent succinate dehydrogenase, is also able to restore a normal $\mathrm{O}_{2}$ consumption (with respiratory control) in digitonin-permeabilized thiamine-deficient cells. Our results therefore suggest that the slowing of the citric acid cycle is the main cause of the biochemical lesion induced by thiamine deficiency as observed in Wernicke's encephalopathy. Key Words: Thiamine deficiency-NecrosisNeuroblastoma cells-Mitochondria-Wernicke's encephalopathy.

J. Neurochem. 65, 2178-2184 (1995).
\end{abstract}

Thiamine diphosphate (TDP), which is the main thiamine compound in the mammalian brain, is a required cofactor for pyruvate and $\alpha$-ketoglutarate dehydrogenases. Therefore, thiamine deficiency should lead to a decreased turnover of the intermediates of the citric acid cycle and, thus, slow down oxidative metabolism (for review, see Butterworth, 1993). The Wernicke-Korsakoff syndrome is a common consequence of thiamine deficiency linked to alcohol abuse in humans (Katzman and Terry, 1983). Wernicke's encephalopathy is characterized by ophthalmoplegia, ataxia, global confusional state, and delusions (Victor et al., 1989). Polyneuropathies are also observed and, at a later stage, there appears a dementia characterized by anterograde amnesia and disorientation (Korsakoff's psychosis; McEntee and Mair, 1990). Although several symptoms of Wernicke's encephalopathy (ophthalmoplegia ) are almost fully reversible after administration of thiamine, others (ataxia) are only partially reversible and the Korsakoff psychosis generally does not respond to thiamine. These thiamine-nonresponsive symptoms probably involve irreversible neuronal lesions. The reasons for the neuronal loss observed in severely thiamine-deficient (TD) brain are probably multiple and involve impaired energy metabolism (Aikawa et al., 1984), acidosis (Hakim and Pappius, 1983), and excitotoxic phenomena (Langlais and Mair, 1990; Hazell et al., 1993).

Since the early work of Peters (for review, see Peters, 1936), it is known that the early symptoms of thiamine deficiency in birds are rapidly reversible on thiamine administration. Moreover, the oxygen consumption was lower in minced brains prepared from avitaminous compared with control pigeons and it was partially restored on addition of thiamine in vitro. Oxidative decarboxylation of pyruvate and $\alpha$-ketoglutarate was decreased in isolated mitochondria prepared from the brains of pyrithiamine-treated rats compared with control animals (Gubler, 1961; Bennett et al., 1966). Normal activity could be rapidly restored after addition of TDP to the mitochondrial preparation (Gubler, 1961). Parker et al. (1984) reported similar observations in mitochondria isolated from TD rat brain. State 3 respiration as well as respiratory control was decreased with substrates such as glutamate, $\alpha$-ketoglutarate, or citrate, but not with succinate, which is metabolized independently of TDP-dependent enzymes. Par-

Received February 6, 1995; revised manuscript received May 15, 1995; accepted May 15, 1995.

Address correspondence and reprint requests to Dr. L. Bettendorff at University of Liège, Laboratory of Neurochemistry, 17 place Dccour, B-4020 Liège, Belgium.

Abbreviations used: CCCP, carbonyl cyanide $m$-chlorophenylhydrazone; TD, thiamine-deficient; TDA, thiamine-deficient and treated with amprolium; TDP, thiamine diphosphate. 
ker et al. (1984) considered that this situation could lead to abnormal glutamate levels, which might favor the appearance of excitotoxic lesions.

In cultured neuroblastoma cells, a reduction of the thiamine concentration in the extracellular medium leads, within days, to an important loss of intracellular thiamine compounds, but the cells keep a nearly normal oxygen consumption (Bettendorff et al., 1995). Addition of amprolium (a thiamine transport inhibitor) to the culture medium leads to a further decrease in intracellular thiamine, decreased respiration, mitochondrial swelling and uncoupling, lactate production, and cell death. The aim of the present study was to investigate the reversibility of those changes after restitution of thiamine to the culture medium.

\section{MATERIALS AND METHODS}

\section{Chemicals}

Thiamine, carbonyl cyanide $m$-chlorophenylhydrazone (CCCP), amprolium, rotenone, digitonin, and oligomycin were purchased from Sigma.

\section{Cell culture}

Neuroblastoma cells were cultured as previously described (Bettendorff and Wins, 1994) in Dulbecco's modilied Eagle's medium (GIBCO, Ghent, Belgium) containing 10) $\mu M$ thiamine and supplemented with $5 \%$ fetal calf serum (GIBCO). TD cells were produced by growing them for at least 2 weeks in a Dulbecco's modified medium devoid of thiamine. Under these conditions, the only thiamine source was the fetal calf serum and its concentration in the medium was $\sim 7 \mathrm{n} M$. To further increase thiamine deprivation, amprolium $(20 \mu M)$ was added to the culture medium of TD cells 4 days before the experiment ("TDA cells"), as described by Bettendorff et al. (1995).

Thiamine derivatives were determined by an HPLC procedure exactly as previously described (Bettendorff et al., 1991). Protein concentrations were determined by the method of Peterson (1977).

Amino acids were extracted from the cells as described by Patel and Hunt (1985) and determined by HPLC (Tapuhi (t) al., 1981).

\section{Oxygen consumption}

Oxygen uptake was measured polarographically in a $2-\mathrm{ml}$ cell at $37^{\circ} \mathrm{C}$ as described by Vayssière et al. (1986), containing $\sim 10-20 \times 10^{6}$ cells in their respective culture media. Digitonin permeabilization was performed as described by Vercesi et al. ( 1991 ).

\section{Electron microscopy}

Monolayer cell cultures were scraped off the dishes and centrifuged at $350 \mathrm{~g}$ for $3 \mathrm{~min}$. Small fragments of the pellet were fixed at $4^{\circ} \mathrm{C}$ in glutaraldehyde $(2.5 \%$ in cacodylate buffer) and then postfixed in $1 \%$ osmium tetroxide solution. The cells were included in Epon. Ultrathin sections mounted on copper grids were stained with uranyl acetate and lead citrate before examination under a Jeol CX 100 II electron microscope at $60 \mathrm{kV}$.

\section{RESULTS AND DISCUSSION}

Figure 1 shows the rate of oxygen consumption by neuroblastoma cells under different experimental con-

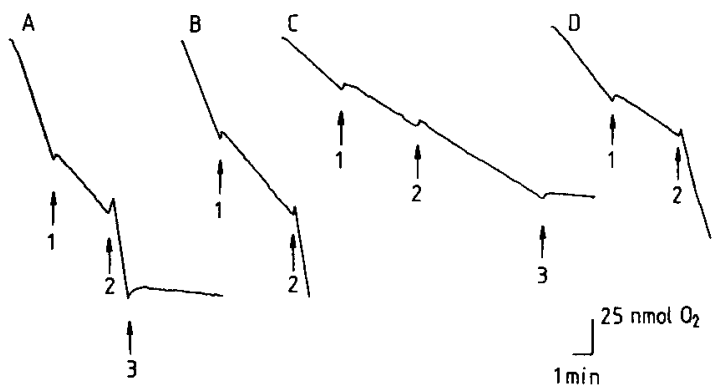

FIG. 1. Polarographic recording of oxygen consumption by neuroblastoma cells in whole culture media containing various amounts of thiamine (A), TD medium (B), TDA medium (C), and TDA cells after addition of $10 \mu \mathrm{M}$ thiamine for $60 \mathrm{~min}$ (D). In the case of TDA cells, amprolium $(20 \mu M)$ was added to the culture days before the experiment. The arrows correspond to the additions of various compounds: 1 , oligomycin $(16 \mu \mathrm{g} / \mathrm{ml}) ; 2, \mathrm{CCCP}$ $(5 \mu M) ; 3, \operatorname{KCN}(1 \mathrm{mM})$.

ditions. The commercial medium always contained 30 $\mathrm{m} M$ glucose, $1 \mathrm{~m} M$ pyruvate, and $4 \mathrm{~m} M$ glutamine. In each case, we first measured the basal oxygen consumption. Then, the rate of $\mathrm{O}_{2}$ consumption was estimated in the presence of added oligomycin, followed by the uncoupler CCCP. In the case of cells grown at a high thiamine concentration $(10 \mu M)$, a reasonably high $\mathrm{O}_{2}$ consumption was measured. As expected, oligomycin decreased oxygen consumption, whereas in the presence of CCCP it was increased about threefold above basal level. Oxygen consumption was completely inhibited after addition of $1 \mathrm{~m} M \mathrm{KCN}$. A similar pattern was observed with cells grown at a lower thiamine concentration $(7 \mathrm{n} M)$. Under those conditions, total intracellular thiamine content was decreased from 210 to $13 \mathrm{pmol} / \mathrm{mg}$ of protein (Bettendorff et al., 1995). To further decrease intracellular thiamine concentration, amprolium $(20 \mu M)$, a competitive inhibitor of thiamine transport, was added to the TD culture medium (TDA) for 4 days. Pyrithiamine is the most potent known thiamine antimetabolite in vivo; but in this study we preferred to use amprolium, though $\sim 30$-fold higher amounts of this compound are required to block thiamine transport (Bettendorff and Wins, 1994). In contrast to pyrithiamine, amprolium cannot be phosphorylated. Thus, no interference with TDP-dependent enzymes is to be expected with amprolium. Under these conditions, the basal respiration was considerably decreased compared with the untreated cells. Furthermore, oligomycin and CCCP were without effect on respiration, suggesting uncoupling of mitochondria. That $\mathrm{KCN}$ (as well as rotenone; not shown) still inhibited oxygen consumption suggests that, even under these severe TD conditions, part of the respiration persists and the most conspicuous effect of thiamine deficiency is the apparent mitochondrial uncoupling. When thiamine was added to the TDA cells $60 \mathrm{~min}$ before the polarographic deter- 


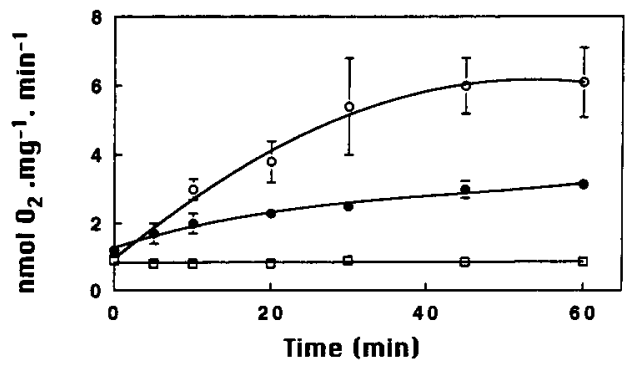

FIG. 2. Time-dependent recovery of oxygen consumption in TDA cells after addition of $10 \mu M$ thiamine. Basal oxygen consumption ( $\bullet$; oxygen consumption in the presence of oligomy$\operatorname{cin}(\square)$ or $\operatorname{CCCP}(O)$. Each point represents the mean $\pm \mathrm{SD}$ value for three experiments except for the points in the presence of oligomycin, which are the mean values of two experiments.

mination of oxygen consumption, a nearly complete recovery of mitochondrial function was observed; i.e., the basal oxygen consumption increased and the usual effects of oligomycin and CCCP returned, suggesting a recoupling of the mitochondria.

Figure 2 shows the time scale of the recovery of mitochondrial respiration. It can be seen that, already 5 min after addition of thiamine, a significant increase in the basal and uncoupled respiration was observed and the effect was complete after $60 \mathrm{~min}$. The respiration in the presence of oligomycin remained unchanged.

Thiamine by itself has no known effect on mitochondrial respiration and the important compound is the cofactor TDP. Thiamine is, however, actively transported into neuroblastoma cells and pyrophosphorylated in the cytoplasm (Bettendorff and Wins, 1994). TDP is then transported into mitochondria (Barile et al., 1990) where it binds to the pyruvate and $\alpha$-ketoglutarate dehydrogenases. A nearly 10 -fold increase in intracellular TDP is observed within $1 \mathrm{~h}$ after addition of thiamine to the cells (Fig. 3). The lag period may be explained by the fact that thiamine pyrophosphokinase is dependent on the intracellular ATP concentration with an exceptionally high $K_{\mathrm{m}}$ of $7 \mathrm{mM}$ (Bettendorff and Wins, 1994). In TDA cells, intracellular ATP concentrations are lowered by $\sim 50 \%$ compared with normal cells (Bettendorff et al., 1995). Thus, the rate of thiamine phosphorylation is slow but increases as mitochondria are producing ATP.

We have previously reported the existence of abnormal mitochondria in TDA cells (Bettendorff et al., 1995). With ongoing thiamine deficiency, the mitochondrial matrix became disorganized and electron translucent; no intact cristae remained visible and some mitochondria became abnormally large. However, we did not observe any significant increase in the number of mitochondria in TDA cells compared with control cells. Mitochondrial respiration in TDA cells was essentially uncoupled and, as a preincubation with thiamine leads to a recoupling of respiration, we wanted to know whether this was accompanied by morphological modifications. Figure $4 \mathrm{a}$ and $\mathrm{b}$ show, with different magnifications, a typical TDA cell with largely electron-translucent mitochondria. The nucleus shows evident signs of chromatin condensation typical of the early abnormalities of necrosis (Wyllie et al., 1980). After $1 \mathrm{~h}$ in the presence of $10 \mu M$ thiamine these abnormalities were essentially reversed (Fig. $4 c$ and d) ; the mitochondria became electron dense and cristae were reformed. The mitochondria resembled those of control cells (Bettendorff et al., 1995). Note that the cell cycle in neuroblastoma cells lasts $\sim 24 \mathrm{~h}$ and, thus, normalization in cell morphology, within $1 \mathrm{~h}$, cannot be explained by the generation of new cells through mitotic division.

The reasons respiratory control is lost in TDA cells are not clear. The link between uncoupling and the disorganization of cristae may appear obvious, as the electrochemical proton gradient tends to dissipate when the inner membranes are damaged; however, the reasons the cristae become disorganized are unclear. We may consider the possibility that the phenomenon is related to the lack of substrates able to donate electrons to the respiratory chain. If this hypothesis is true, the direct addition of a permeant substrate should reverse uncoupling in a manner analogous to thiamine addition, maybe even faster. As shown in Fig. 5, the addition of succinate to cells permeabilized with digitonin indeed restored coupled respiration. After addition of digitonin to TDA cells, the oxygen consumption gradually decreased, as a result of dilution of the remaining substrates. Addition of succinate increased the respiration up to sixfold in some experiments. This $\mathrm{O}_{2}$ consumption was slightly inhibited by oligomycin and increased by CCCP as expected. The uncoupled respiratory control, i.e., the ratio of $\mathrm{O}_{2}$ consumption in the presence of CCCP to the $\mathrm{O}_{2}$ consumption in the presence of oligomycin, was $1.8 \pm 0.5$. This uncoupled respiratory control value was not significantly higher when normal (instead of TDA) cells were used. Rotenone, an inhibitor of respiratory chain complex I, was without effect on $\mathrm{O}_{2}$ consumption after succinate addition, but antimycin, an inhibitor of complex III, nearly completely inhibited oxygen consumption. This sug-

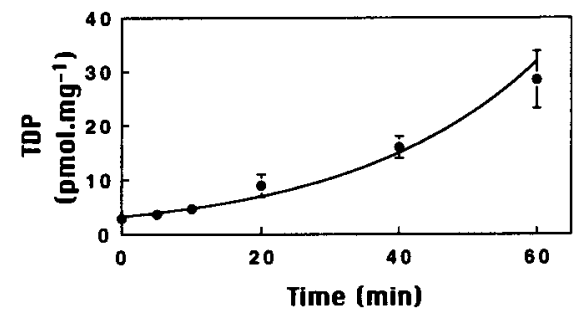

FIG. 3. Time-dependent recovery of intracellular TDP content in TDA cells after addition of $10 \mu M$ thiamine. Each point represents the mean $\pm S D$ for three experiments. 
FIG. 4. Electron micrographs of TDA cells $(\mathbf{a}, \mathbf{b})$ and TDA cells exposed to $10 \mu \mathrm{M}$ thiamine for $1 \mathrm{~h}(\mathbf{c}, \mathbf{d})$. Magnification bars: 1 $\mu \mathrm{m}$ in (a) and (c); $0.2 \mu \mathrm{m}$ in (b) and (d).
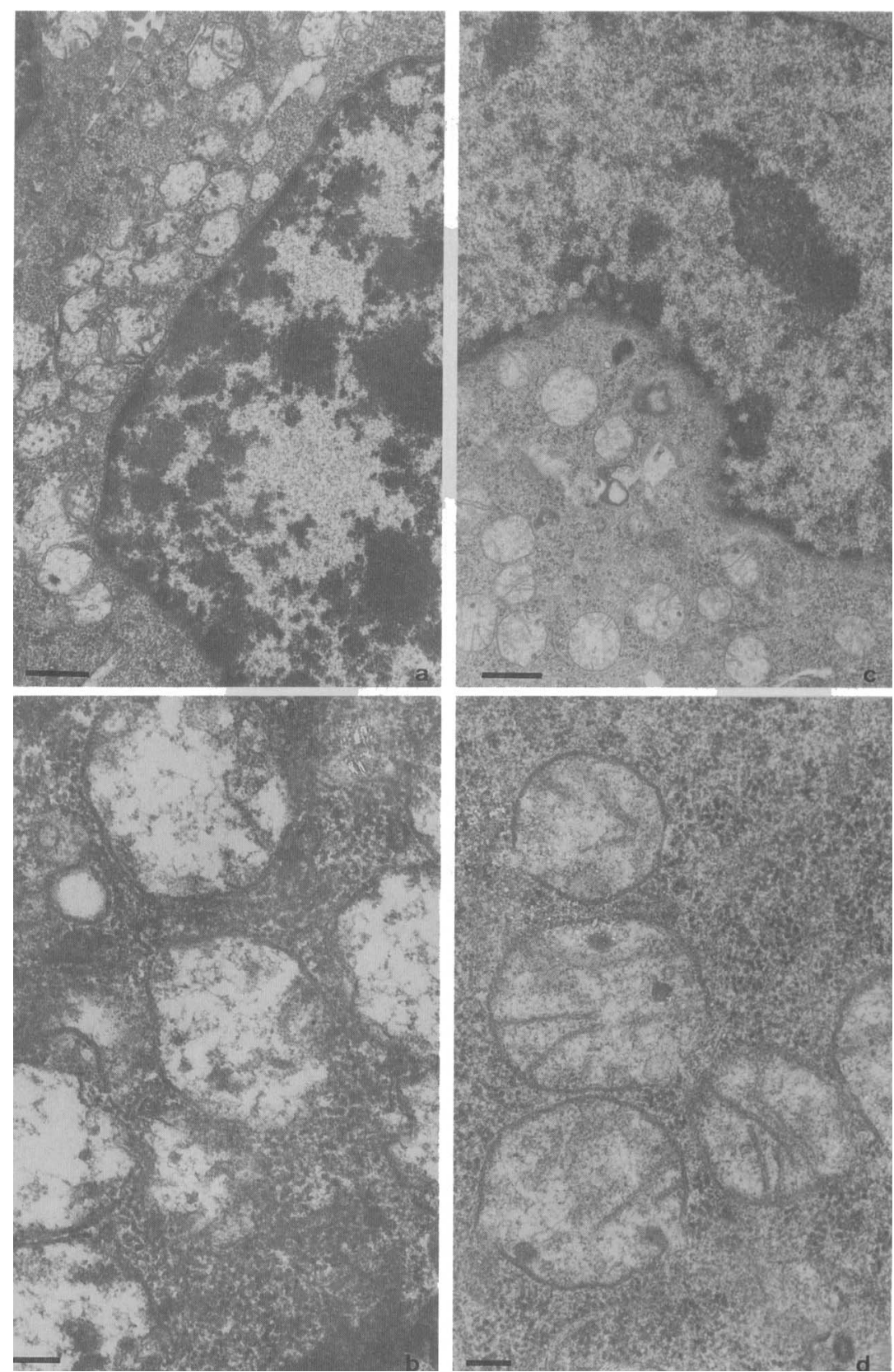

gests that, under these conditions, the respiration is indeed sustained by succinate. In TDA cells, at least some of the mitochondria remain functional, and the apparent uncoupling (Fig. 1C) appears to be linked to the lack of oxidizable substrates. A direct consequence of this lack of reducing substrates is that at least complexes III and IV will remain completely oxidized for a long time. This may somehow lead to disorganization of cristae, but the molecular mechanisms involved, if any, remain unknown. Mitochondrial swelling with rupture of cristae has long been known to occur after treatment with ascorbate, ferrous ions (Hunter et al., 1963 ), or after treatments that favor lipid peroxidation (Shigenaga et al., 1994). Swelling and inner membrane damage were also observed after treatment of mitochondria by thiol-blocking alkylating agents (Lê Quôc and Lê Quôc, 1985), glutathione deficiency (Jain et al., 1991), and deficiency in an enzyme involved in cardiolipin synthesis (Ohtsuka et al., 1993). In all cases described so far, however, there is no evidence that the respiratory chain is involved.

As thiamine deficiency has been reported to affect 


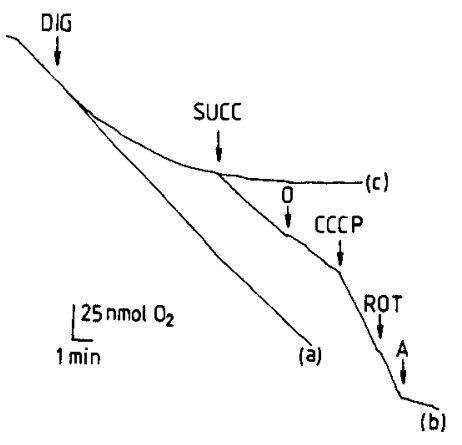

FIG. 5. Respiration in digitonin-permeabilized TDA cells. The cells were sedimented and the culture medium replaced by the test medium containing $125 \mathrm{mM}$ sucrose, $65 \mathrm{mM} \mathrm{KCl}, 10 \mathrm{mM}$ Tris- $\mathrm{HCl}(\mathrm{pH} 7.2), 1 \mathrm{mM} \mathrm{MgCl}, 0.33 \mathrm{mM}$ EGTA, $2.5 \mathrm{mM}$ $\mathrm{KH}_{2} \mathrm{PO}_{4}$, and $2.5 \mathrm{mM}$ ADP. Trace (a) shows the oxygen consumption without addition. In traces (b) and (c), $50 \mu \mathrm{M}$ digitonin (DIG) was added. Other additions (trace b) are $16 \mu \mathrm{g} / \mathrm{ml}$ oligomycin (O), $50 \mathrm{nM}$ CCCP, $2 \mu M$ rotenone (ROT), and $2 \mu \mathrm{g} / \mathrm{ml}$ antimycin (A).

amino acid metabolism in rat brain (Butterworth and Héroux, 1989; Page et al., 1989; Butterworth, 1993), we determined the concentrations of intracellular amino acids in our cells (Table 1 ). In control cells, the amino acid concentrations were close to those previously reported in cultured neurons and astrocytes (Patel and Hunt, 1985). As expected, glutamate was the most abundant amino acid. We were unable to detect any GABA in our cells, an observation that is important for the interpretation of our results as this means that they lack glutamate decarboxylase, an enzyme specific for GABAergic neurons (Martin, 1986). This implies that, in our cells, no succinate can be formed through the GABA shunt when $\alpha$-ketoglutarate dehydrogenase is inactive due to lack of TDP. Thiamine deficiency increases the metabolic flux through this pathway (Page et al., 1989) and it can thus be expected that cells with a functionally intact GABA shunt would be more resistant to thiamine deficiency than cells that do not possess this pathway. However, it has been shown that glutamate decarboxylase activity and GABA levels reversibly decrease in the brain of TD rats (Héroux and Butterworth, 1988), which may cause disturbances in GABAergic neurotransmission and add to the reversible symptoms caused by thiamine deficiency in brain.

Aspartate, glycine, and alanine levels were significantly increased in TD compared with control cells. That alanine is increased suggests that pyruvate dehydrogenase is already partially inhibited in these cells; i.e., pyruvate, instead of entering the citric acid cycle, is partially transaminated to alanine. Addition of amprolium leads to a large increase in intracellular glutamine and glutamate concentrations. In neurons, $\alpha$-ketoglutarate and glutamate rapidly and reversibly equilibrate in parallel with oxaloacetate and aspartate, as a result of very fast transamination (Erecińska et al., 1993 ). In brain cells, glutamate dehydrogenase activity is probably less important than transamination in the formation of $\alpha$-ketoglutarate from glutamate (McCarthy and Tipton, 1983). Indeed, glutamate dehydrogenase is more likely to catalyze the reverse reaction, especially in the presence of high $\mathrm{NH}_{4}{ }^{+}$concentrations. In intact brain, glucose is practically the only substrate crossing the blood-brain barrier and, hence, glutamate is essentially formed from $\alpha$-ketoglutarate derived from glucose. This might explain that in the TD brain glutamate levels are decreased (Plaitakis et al., 1979; Butterworth and Héroux, 1989). In our cells, however, glutamine is taken up from the culture medium and is the direct source of glutamate. In TDA cells, where $\alpha$ ketoglutarate dehydrogenase is probably strongly inhibited, $\alpha$-ketoglutarate will accumulate (especially in the absence of the GABA shunt) and the glutamate formed from glutamine is no longer oxidized via the citric acid circle. There is no parallel increase in aspartate concentration presumably because no oxaloacetate can be formed.

Addition of thiamine to the cells leads, within $1 \mathrm{~h}$, to a significant decrease in intracellular glutamate level. This would be in agreement with a recovery of $\alpha$-ketoglutarate dehydrogenase activity and oxidative metabolism. The respiration in the presence of thiamine is nearly completely blocked by rotenone (an inhibitor of NADH dehydrogenase) in agreement with this observation.

One of the most puzzling observations in thiamine deficiency, in animal models as well as in human pathology, is that the early symptoms are so rapidly reversed on thiamine administration (Butterworth, 1993 ). This is the "biochemical lesion" described by Peters (1936). Prolonged thiamine deficiency, however, leads to irreversible histological lesions with neuronal death. In this and our previous work (Bettendorff et al., 1995), we show that, in cultured neuroblastoma cells, severe thiamine deficiency leads to a decrease in the rate of respiration, to mitochondrial uncoupling,

TABLE 1. Amino acid contents of neuroblastoma cells under various conditions of thiamine deficiency

\begin{tabular}{lccccc}
\hline & \multicolumn{5}{c}{ nmol/mg of protein } \\
\cline { 2 - 6 } & Gln & Asp & Glu & Gly & Ala \\
\hline Control & $4.2 \pm 0.9$ & $5.2 \pm 0.4$ & $65 \pm 8$ & $43 \pm 5$ & $23 \pm 1$ \\
TD & $3.4 \pm 2.0$ & $12 \pm 3$ & $56 \pm 13$ & $66 \pm 2$ & $41 \pm 4$ \\
TDA & $21 \pm 8$ & $9.4 \pm 1$ & $165 \pm 42$ & $83 \pm 7$ & $22 \pm 3$ \\
TDA + & & & & & \\
$\quad$ thiamine & $58 \pm 13$ & $10 \pm 3$ & $118 \pm 15$ & $61 \pm 1$ & $22 \pm 3$ \\
\hline
\end{tabular}

Amino acids were determined by HPLC according to the method of Tapuhi et al. (1981). The last group represents TDA cells incubated in the presence of thiamine $(10 \mu M)$ for $1 \mathrm{~h}$. In each case, the culture medium contained $0.4 \mathrm{~m} M$ glycine and $4 \mathrm{~m} M$ glutamine. Mean $\pm \mathrm{SD}$ values for three experiments. 
and to morphological abnormalities corresponding to the early symptoms of necrosis (Wyllie et al., 1980). ATP concentrations are decreased, lactate production increases, the cells become depolarized, and cell mortality increases (Bettendorff et al., 1995). The most remarkable property shown in the present study is that those cells that survive long enough under severe deficiency respond rapidly to thiamine treatment; i.e., normal respiration as well as normal cell and mitochondrial morphology are recovered within $1 \mathrm{~h}$. These results suggest that the biochemical lesion observed in thiamine deficiency is the result of an inability of the cells to oxidize substrates. Energy failure leads to a cellular collapse, which, if not treated, results in necrosis and cell death.

In a recent report, Zhang et al. (1995) demonstrated the existence of disintegrating mitochondria and chromatin clumping in degenerating neurons of diencephalic nuclei in pyrithiamine-treated rats. These results suggest that, in brain, mechanisms similar to those described here for cultured neuroblastoma cells may be operating, except that additional phenomena such ats excitotoxicity might contribute to the selective vulnerability of certain brain regions in pyrithiamine rats (Langlais and Mair, 1990; Hazell et al., 1993; Langlais and Zhang, 1993). So far, however, there is no evidence that excitotoxic phenomena are important during the acute, reversible phase of thiamine deficiency (Wernicke's encephalopathy).

Acknowledgment: We thank the Belgian National Funds for Scientific Research (FNRS) for a grant to L.B. P.W. is Research Associate at the FNRS.

\section{REFERENCES}

Aikawa A. H., Watanabe I. S., Furuse T., Iwasaki Y., Satoyoshi E., Sumi T., and Morojii T. (1984) Low energy levels in thiaminedeficient encephalopathy. J. Neuropathol. Exp. Neurol. 43, $276-287$.

Batrile M., Passarella S., and Quagliariello E. (1990) Thiamine pyrophosphate uptake into isolated rat liver mitochondria. Arch. Biochem. Biophys. 280, 352-357.

Bennett C. D. Jones J. H., and Nelson J. (1966) The effects of thiamine deficiency on the metabolism of the brain: I. Oxidation of various substrates in vitro by the liver and brain of normal and pyrithiamine-fed rats. J. Neurochem. 13, 449-459.

Bettendorff L. and Wins P. (1994) Mechanism of thiamine transport in neuroblastoma cells. Inhibition of a high affinity carrier by sodium channel activators and dependence of thiamine uptake on membrane potential and intracellular ATP. J. Biol. Chem. 269, $14379-14385$.

Beltendorff L., Peeters M., Jouan C., Wins P., and Schoffeniels E. (1991) Determination of thiamin and its phosphate esters in cultured neurons and astrocytes using an ion-pair reversed phase high-performance liquid chromatographic method. Anal. Bio(hem. 198, 52-59.

Betlendorff L., Goessens G., Sluse F., Wins P., Bureau M., Laschet J., and Grisar T. (1995) Thiamine deficiency in cultured neuroblastoma cells: effect on mitochondrial function and peripheral benzodiazepine receptors. J. Neurochem. 64, 2013-2021.

Butterworth R. F. ( 1993 ) Pathophysiological mechanisms responsible for the reversible (thiamine-responsive) and irreversible (thiamine non-responsive) neurological symptoms of Wernicke's encephalopathy. Drug Alcohol Rev. 12, 315-322.

Butterworth R. F. and Héroux M. (1989) Effect of pyrithiamine treatment and subsequent thiamine rehabilitation on regional cerebral amino acids and thiamine-dependent enzymes. $J$. Neurochem. 52, 1079-1084.

Erecińska M., Pleasure D., Nelson D., Nissim I., and Yudkoff M. (1993) Cerebral aspartate utilization: near-cquilibrium relationships in aspartate aminotransferase reaction. I. Neurochem. 60, $1696-1706$.

Gubler C. J. (1961) Studies on the physiological functions of thiamine. I. The effects of thiamine deficiency and thiamine antagonists on the oxidation of $\alpha$-keto acids by rat tissues. $J$, Biol. Chem. 236, 3112--3120.

Haas R. H. (1988) Thiamin and the brain. Annu. Rev. Nutr. 8, 483515.

Hakim A. M. and Pappius H. M. (1983) Sequence of metabolic, clinical and histological events in experimental thiamine deficiency. Ann. Neurol. 13, 365-375.

Hazell A. S., Butterworth R. F., and Hakim A. M. (1993) Cerebral vulnerability is associated with selective increase in extracellular glutamate concentrations in experimental thiamine deficiency. J. Neurochem. 61, 1155-1158.

Héroux B. and Butterworth R. F. ( 1988 ) Reversible alterations of cerebral $\gamma$-aminobutyric acid in pyrithiamine-treated rats: implications for the pathogenesis of Wernicke's encephalopathy. $J$. Neurochem. 51, 1221-1226.

Hunter F. E. Jr., Gebicki J. M., Hoffsten P. E., Weinstein J., and Scott A. (1963) Swelling and lysis of rat liver mitochondria induced by ferrous ions. J. Biol. Chem. 238, 828-835.

Jain A., Mårtensson J., Stole E., Auld P. A. M.. and Meister A. (1991) Glutathione deficiency leads to mitochondrial damage in brain. Proc. Natl. Acad. Sci. USA 88, 1913-1917.

Katzman R. and Terry R. D. (1983) The Neurology of Aging. F. A. Davis, Philadelphia.

Langlais P. J. and Mair R. G. (1990) Protective effects of the glutamate antagonists $\mathrm{MK}-8() 1$ on pyrithiaminc-induced lesions and amino acid changes in rat brain. J. Neurosci. 10, 1664-1674.

Langlais P. J. and Zhang S. X. (1993) Extracellular glutamate is increased in thalamus during thiamine deficiency-induced lesions and is blocked by MK-801. J. Neurochem. 61, 21752182.

Lề-Quôc K. and Lê-Quôc D. ( 1985 ) Crucial role of sulfhydryl groups in the mitochondrial inner membrane structure. J. Biol. Chem. 260, 7422-7428.

Martin D. L. ( 1986 ) Brain glutamate decarboxylase, in Neurotransmitter Enzymes (Boulton A. A., Baker G. B., and Yu P. H., eds), pp. 261-388. Humana Press, Clifton, New Jersey.

McCarthy A. D. and Tipton K. F. ( 1983 ) Glutamate dehydrogenase, in Glatamine, Glutamate, and GABA in the Nervous System (Hertz L... Kvamme E., McGeer E. G., and Schousboe A., eds), pp. 19-32. Alan R. Liss, New York.

McEntee W. J. and Mair R. G. ( 1990) The Korsakoff syndrome: a neurochemical perspective. Trends Neurosci. 13, 340-344.

Ohtsuka T., Nishijima M., Suzuki K., and Akamatsu Y. (1993) Mitochondrial dysfunction of a cultured Chinese hamster ovary cell mutant deficient in cardiolipin. J. Biol. Chem. 268, 2291422919.

Page M. G., Ankoma-Sey V., Coulson W. F., and Benders D. A. (1989) Brain glutamate and $\gamma$-aminobutyrate (GABA) metabolism in thiamine-deficient rats. Br. J. Nutr. 62, 245-253.

Parker W. D. Jr., Haas R., Stumpf D. A., Parks J., Eguren L. A., and Jackson C. (1984) Brain mitochondrial metabolism in experimental thiamine deficiency. Neurology 34, 1477-1481.

Patel A. J. and Hunt A. (1985) Concentration of free amino acids in primary cultures of neurones and astrocytes. J. Neurochem. 44, $1816-1821$.

Peters R. A. ( 1936 ) The biochemical lesion in vitamin B 1 deficiency. Application of modern biochemical analysis in its diagnosis. Lancet 1, 1161-1164. 
Peterson G. L. ( 1977 ) A simplification of the protein assay method of Lowry et al. which is more generally applicable. Anal. Biochem. 83, 346-356

Plaitakis A., Nicklas W. J., and Berl S. (1979) Alterations in uptake and metabolism of aspartate and glutamate in brain of thiamine deficient animals. Brain Res. 171, 489-502.

Shigenaga M. K., Hagen T. M., and Ames B. N. (1994) Oxidative damage and mitochondrial decay in aging. Proc. Natl. Acad. Sci. USA 91, 10771-10778.

Tapuhi V., Miller N., and Karger B. L. (1981) Practical considerations in the chiral separation of Dns-amino-acids by reversedphase liquid chromatography using metal chelate additives. $J$. Chromatogr. 205, 325-337.

Vayssiere J. L., Larcher J. C., Berthelot F., Benlot C., Gros F., and Croizat B. ( 1986 ) Effects on mitochondrial metabolism of
CCA, one inducer of neuroblastoma differentiation. Biochem. Biophys. Res. Commun. 140, 789-796.

Vercesi A. E., Bernardes C. F., Hoffmann M. E., Gadelha F. R., and Docampo R. (1991) Digitonin permeabilization does not affect mitochondrial function and allows the determination of the mitochondrial membrane potential of Trypanosoma cruzi in situ. J. Biol. Chem. 266, $14431-14434$.

Victor M., Adams R. D., and Collins G. H. (1989) The WernickeKorsakoff Syndrome. F. A. Davis, Philadelphia.

Wyllie A. H., Kerr J. F. R., and Currie A. R. (1980) Cell death: the significance of apoptosis. Int. Rev. Cytol. 68, 251-306.

Zhang S. X., Weilersbacher G. S., Henderson S. W., Corso T., Olney J. W., and Langlais P. J. (1995) Excitotoxic cytopathology, progression, and reversibility of thiamine deficiency-induced diencephalic lesions. J. Neuropathol. Exp. Neurol. 54, 255267. 